\title{
Influence of curing mode of resin luting cements on bond strength to dentin
}

\author{
Andreia Assis Carvalho ${ }^{1}$, Ariovaldo Stefani², Wladimir Franco de Sá Barbosa ${ }^{3}$, Lawrence Gonzaga Lopes ${ }^{4}$, \\ Marcelo Giannini ${ }^{5}$
}

${ }^{1}$ Mestre em Clínica Odontológica, Universidade Federal de Goiás - UFG, Department of Prevention and Oral Rehabilitation, Goiânia, Goiás, Brazil, Address:

Department of Prevention and Oral Rehabilitation - School of Dentistry, Federal University of Goiás, Praça Universitária, s/n, Faculdade de Odontologia,

Setor Universitário, Goiânia-GO, Brazil, CEP 74605220

${ }^{2}$ Mestre em Prótese, São Leopoldo Mandic - SLMANDIC, Department of Prosthesis, Campinas, SP, Brazil, Address: Rua Borges Lagoa, 1065, cj 139, Vila

Clementino, São Paulo, SP, Brazil. CEP 04038-032

${ }^{3}$ Mestre em Clínica Odontológica, Universidade do Estado do Amazonas - UEA, Department of Dentistry, Manaus, AM, Brazil, Address: State University of Amazonas, School of Health Sciences, Odontologia, Av. Carvalho Leal, 1777, Cachoeirinha, Manaus, AM, Brazil. CEP 69065-001

${ }^{4}$ Doutor e Mestre em Dentística, Universidade Federal de Goiás - UFG, Department of Prevention and Oral Rehabilitation, Goiânia, Goiás, Brazil, Address:

Department of Prevention and Oral Rehabilitation - School of Dentistry, Federal University of Goiás - Praça Universitária, s/n, Faculdade de Odontologia,

Setor Universitário, Goiânia-GO, Brazil, CEP 74605220

${ }^{5}$ Doutor e Mestre em Clínica Odontológica, Universidade Estadual de Campinas - UNICAMP, Associate Professor, Department of Restorative Dentistry, Piracicaba Dental School, Piracicaba, SP, Brazil, Address: Department of Restorative Dentistry - Piracicaba Dental School - Campinas State University - Av.

Limeira, 901 - Piracicaba, SP, Brazil, CEP 13414-903018

Received for publication: December 11, 2016 Accepted: May 17, 2017

Correspondence to: Dr. Marcelo Giannini Department of Restorative Dentistry Piracicaba Dental School Campinas State University Av. Limeira, 901 - Piracicaba, SP, Brazil CEP 13414-903018

e-mail: giannini@fop.unicamp.br phone: $+55-19-34125338$

fax: $+55-19-34125218$

\begin{abstract}
Self-adhesive, dual-polymerizing resin cements require no treatment to the prepared tooth surfaces before cementation. Aim: The aim of this study was to evaluate the influence of curing mode on bond strength (BS) of three cementing systems to bovine dentin. Methods: The buccal enamel surfaces of 50 bovine incisors were removed to expose dentin and to flat the surface. The teeth were divided into five groups $(n=10)$, which consisted of two resin cements (Multilink and Clearfil SA Cement) that were tested in dual- (halogen light for $40 \mathrm{~s}$ ) and self-cured modes, and a control (RelyXARC). Two cylinders of resin cements $(1.0 \mathrm{~mm} \times 0.75 \mathrm{~mm})$ were prepared on each bonded dentin surface. After $24 \mathrm{~h}$ at $370 \mathrm{C}$, resin cylinders were subjected to micro-shear testing in a universal testing machine (4411/Instron - $0.5 \mathrm{~mm} / \mathrm{min})$. Data were statistically analyzed by two-way ANOVA, Tukey and Dunnett's test (5\%). Results: Multilink showed higher BS than those observed on Clearfil SA. Light-curing resulted in higher BS for both Multilink and Clearfil SA. When Multilink was light-cured, no significant difference on BS was demonstrated between it and RelyX ARC. Conclusions: The highest BS values were obtained in control group and light-cured Multilink resin cement.
\end{abstract}

Keywords: Tensile Strength. Adhesives. Dentin.

\section{Introduction}

Resin cements (RC) are low viscous composite resins used to fix indirect restorations and to promote sealing between restoration and tooth substrates ${ }^{1}$. The longevity of indirect restorations is directly connected with the effectiveness of the bond between dental tissues and $\mathrm{RC}^{2}$.

RC can be classified as light-cured, self/auto-cured or dual-cured based on polymerization mode, without considering the dentin pretreatment method ${ }^{3} . \mathrm{RC}$ are produced in dual-polymerized formulations, which are indicated for restorations with 
high opacity or sufficient thickness to inhibit light energy from tip of curing unit to the $\mathrm{RC}$ or other clinical scenarios when the light is significantly attenuated ${ }^{4}$.

According to the treatment of dental tissue, the $\mathrm{RC}$ are classified: total-etch, self-etch or self-adhesive ${ }^{2}$. At present, all $\mathrm{RC}$ are based on the use of either an etch-and-rise or of a selfadhesive, however, application in stages technology is complex and considerably technology sensitive and thus may affect bonding efficiency ${ }^{5}$. The new self-adhesive, dual-polymerizing RC require no separate acid etching, priming or bonding resin applications to the prepared tooth surfaces before ${ }^{6}$. However, little is known about the effectiveness of these new cements regarding the bonding to mineralized dental tissues when light-cured or with no curing light exposure. The purpose of this study was to evaluate the influence of curing mode on bond strength of RC to bovine dentin, using two resin cements that were either light polymerized or allowed to autopolymerize in the absence of light. Furthermore, the site morphology of bond failures was analyzed to classify them according to the fracture mode. The null hypotheses tested were that light-curing has no effect in bond strength values and no experimental group would reach the BS of control group.

\section{Materials and methods}

The materials (cementing systems: resin cement and bonding agent) used in this study and their compositions are described in Table 1. The RelyX ARC (3M ESPE, St. Paul, MN, USA) that is dual-cured RC was used in combination with a three-steps etchand-rinse adhesive (Scotchbond Multipurpose, 3M ESPE, St. Paul, MN, USA) and considered the control in this study. Multilink is a dual-cured $\mathrm{RC}$ used in combination with a self-etching primer (mixed Multilink Primer A/B) (Ivoclar Vivadent, Schaan, Liechtenstein). Clearfil SA Cement (Kuraray Noritake Dental Inc., Kurashiki, Japan) is dual-cured self-adhesive RC, which does not require a previous bonding agent application.

Fifty freshly extracted bovine incisors stored at $60 \mathrm{C}$ were used in this study. The roots and labial enamel were removed using a diamond disk (Isomet, Buehler, Evanstone, IL, USA) under water-cooling. The dentin surfaces were wet-abraded with 600 -grit $\mathrm{SiC}$ paper (Carborundum, Vinhedo, SP, Brazil) to expose a flat dentin. Teeth were randomly divided into five groups $(n=10)$ :

Group 1: Primer A and B / Multilink (dual-cured mode / light- and self-curing)

Group 2: Primer A and B / Multilink (only self-cured mode)

Group 3: Clearfil SA Cement (dual-cured mode / light- and self-curing)

Group 4: Clearfil SA Cement (only self-cured mode)

Control: Scotchbond Multipurpose / RelyX ARC (regular use with light-activation of adhesive and resin cement).

The RC and bonding agents were applied according to the manufacturer's instructions and all light-curing procedures were performed with the Optilux 500 (Demetron, Kerr, Orange, CA, USA) light-curing unit $\left(650 \mathrm{~mW} / \mathrm{cm}^{2}\right)$. The methodology developed by Shimada et al. ${ }^{7}$ (2002) was used to prepare specimens for the micro-shear test. Two cylindrical translucent molds (Tygon tubing, TYG-030, Saint-Gobain Performance Plastics, Aurora, OH,
USA) were positioned over dentin and freshly mixed dual-cure RC (Multilink, Clearfil SA Cement or RelyX ARC) were placed in the molds to fill their internal volume and light activated for $40 \mathrm{~s}$ or not, depending on the experimental group.

Table 1 - Compositions of the resin luting cements and bonding agents used.

\begin{tabular}{ll}
\hline Material (Resin Cement or Bonding Agent) & Composition (lot number) \\
\hline Multilink & Multilink Automix: dimethacrylate, \\
& 2- hydroxyethyl- methacrylate, barium \\
& glass, ytterbium trifluoride, spheroid \\
& mixed oxide. (LM0577) \\
& Multilink Primer A: aqueous solution of \\
& initiators. (N30020) \\
& Multilink Primer B: 2- hydroxyethyl- \\
& methacrylate, phosphonic acid acrylate \\
& monomers. (N38177) \\
\hline Clearfil SA Cement & Bisphenol A diglycidylmethacrylate, \\
& sodium fluoride, triethyleneglycol di- \\
& methacrylate, 10-methacryloyloxydecyl \\
& dihydrogen phosphate, hydrophobic \\
& aromatic dimethacrylate, hydrophobic \\
& aliphatic dimethacrylate, silanated \\
& barium glass filler, silanated colloidal \\
& silica, dl-camphorquinone, initiators, \\
& accelerators, catalysts, pigments, \\
& others. (0004AB) \\
\hline Paste A: silane-treated ceramic, trieth- \\
yleneglycol dimethacrylate, polymer. \\
(GE9JG) \\
Paste B: silane-treated ceramic, tetra- \\
thyleneglycol dimethacrylate, bis-GMA, \\
silane treated silica, functionalized \\
dimethacrylate polymer. \\
\hline Primer: water, 2- hydroxyethyl- methac- \\
rylate, copolymer of acrylic and itaconic \\
acids. (9CC) \\
Bond: bis-GMA, 2- hydroxyethyl- meth- \\
acrylate, initiators. (9RL) \\
\\
\hline Scotchbond Multipurpose
\end{tabular}

Teeth were stored in $100 \%$ humid environment at $37^{\circ} \mathrm{C}$ for $24 \mathrm{~h}$. The tube molds were removed to expose the RC cylinders ( $1.0 \mathrm{~mm}$ high by $0.75 \mathrm{~mm}$ diameter) bonded to the dentin surface, thus, two bonded small RC cylinders were obtained for each dental fragment. Prior to testing, all resin cylinders were checked under stereomicroscope (30x, Meiji Techno Co. LTD., Saitama, Japan) for bonding defects and RC flashing.

Each tooth was attached to the testing device with cyanoacrylate glue (Zapit, DVA, Corona, CA, USA) and tested in a universal testing machine (EZ-test 500, Shimadzu Co, Tokyo, Japan). A shear load was applied at the base of the RC cylinder with a thin wire $(0.20 \mathrm{~mm}$ diameter $)$ at a crosshead speed of 1.0 $\mathrm{mm} / \mathrm{min}$ until failure ${ }^{8}$. The shear bond strength were calculated and expressed in $\mathrm{MPa}$. A bond strength value was then calculated for each tooth by averaging the values of two cylinders from that tooth. Exploratory analysis of data regarding normality was 
performed by Kolmogorov-Smirnov test. Bond strength data were analyzed statistically by two-way (cement $\mathrm{X}$ activation mode) analysis of variance (ANOVA), Tukey and Dunnett's tests at the $5 \%$ level of significance.

After testing, the teeth were mounted on aluminum stubs, gold-sputter coated (SCD 050, Baltec, Vaduz, Liechtenstein) and observed under high vacuum with a scanning electron microscope (JSM-5600LV, Jeol, Tokyo, Japan). Photomicrographs of representative areas of the fractured surfaces were taken at 100X magnification for fracture pattern evaluation, which was classified as (1) adhesive along the dentin, (2) cohesive within the RC and (3) mixed when simultaneously exhibiting dentin surface, remnants of RC and/or adhesive and cohesive failure within the dentin.

\section{Results}

The bond strength means (and standard deviation) of the five study groups are shown in Table 2. The Tukey's test for multiple comparisons among four experimental groups (excluding the RelyX ARC control group) showed that the highest dentin bond strength was obtained when Multilink was light-cured $(\mathrm{p}<0.05)$. The bond strengths of Multilink RC were always higher than those obtained with Clearfil SA Cement, independent on the cure mode tested $(\mathrm{p}<0.05)$. The light activation improved the dentin bond strength for both RC ( $<<0.05)$ (Table 3). Dunnett's test compared control and experimental groups and indicated that only the light-activated Multilink group did not differ statistically from the control $(\mathrm{p}=0.836)$ (Table 4).

Table 2 - The bond strengths means (and standard deviation) in $\mathrm{MPa}$ for the resin cements tested.

\begin{tabular}{lcc}
\hline Group & mean & sd \\
\hline Multilink Light-cured & $29.5 \mathrm{~A}^{*}$ & 7.9 \\
\hline Multilink Self-cured & $21.3 \mathrm{~B}$ & 6.4 \\
\hline Clearfil Light-cured & $10.8 \mathrm{C}$ & 3.1 \\
\hline Clearfil Self-cured & $3.0 \mathrm{D}$ & 3.9 \\
Rely X F Light-cured (control) & 31.5 & 5.5 \\
\hline
\end{tabular}

* Significant difference from the control by Dunnett's test $(\mathrm{p}<0.05)$.

Table 3 - Tukey`s test for multiple comparisons among experimental groups, except for RelyX ARC.

\begin{tabular}{lc}
\hline Comparison & $p$ \\
\hline Multilink Light-cured x Multilink Self-cured & $0.014^{*}$ \\
\hline Multilink Light-cured $x$ Clearfil Light-cured & $<0.001^{*}$ \\
\hline Multilink Light-cured $x$ Clearfil Self-cured & $<0.001^{*}$ \\
\hline Multilink Self-cured x Clearfil Light-cured & $0.001^{*}$ \\
Multilink Self-cured x Clearfil Self-cured & $<0.001^{*}$ \\
\hline Clearfil Light-cured x Clearfil Self-cured & $0.021^{*}$ \\
\hline
\end{tabular}

\footnotetext{
* statistically significant difference $(\mathrm{p}<0.05)$
}

Table 4 - Dunnett's test for comparison between the control group (RelyX ARC) and experimental groups.

\begin{tabular}{lc}
\hline Comparison & $p$ \\
\hline RelyX ARC Light-cured x Multilink Light-cured & $0.836 \mathrm{~ns}$ \\
\hline RelyX ARC Light-cured x Multilink Self-cured & $0.001^{*}$ \\
\hline RelyX ARC Light-cured x Clearfil Light-cured & $<0.001^{*}$ \\
\hline RelyX ARC Light-cured x Clearfil Self-cured & $<0.001^{*}$ \\
* statistically significant difference $(\mathrm{p}<0.05)$. & \\
ns: no statistically significant difference. &
\end{tabular}

Scanning electron microscopy (SEM) examination of fractured interfaces showed variations among groups. Multilink produced mixed fractures, which involved adhesive failure along dentin surface, remnants of RC and/or adhesive and cohesive failure within the dentin (Figures 1 and 2). For Clearfil SA Cement, cohesive fractures within $\mathrm{RC}$ were predominant when the materials were not light exposed (Figure 3), while the adhesive failures along the dentin were seen when light-cured (Figure 4). Adhesive failures were predominant for RelyX ARC (Figure 5).

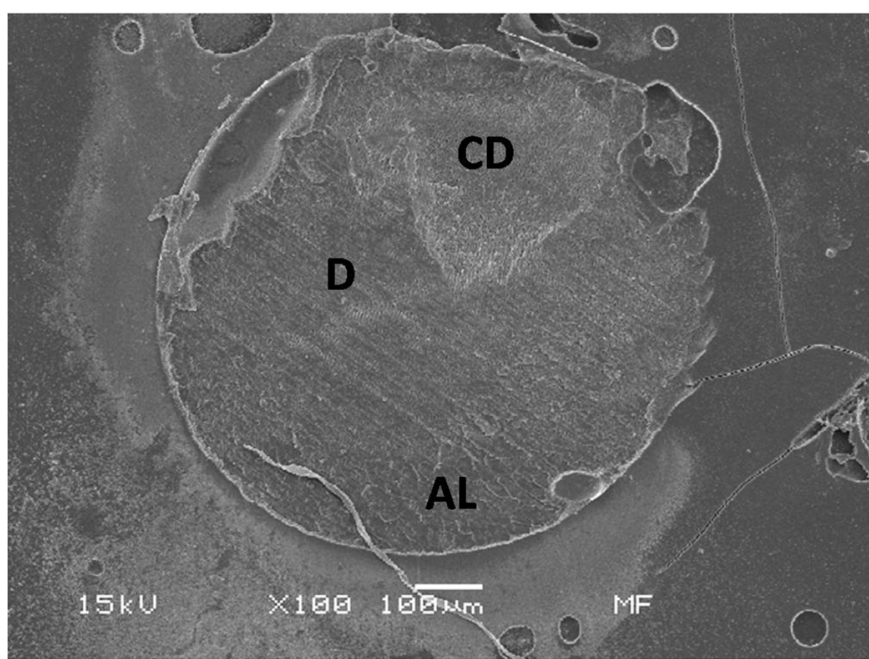

Fig. 1 - SEM photomicrograph illustrating a mixed fracture for Multilink (light-cured). Fracture within the dentin (CD) and failure between dentin and resin cement (AL: adhesive layer, D: dentin).

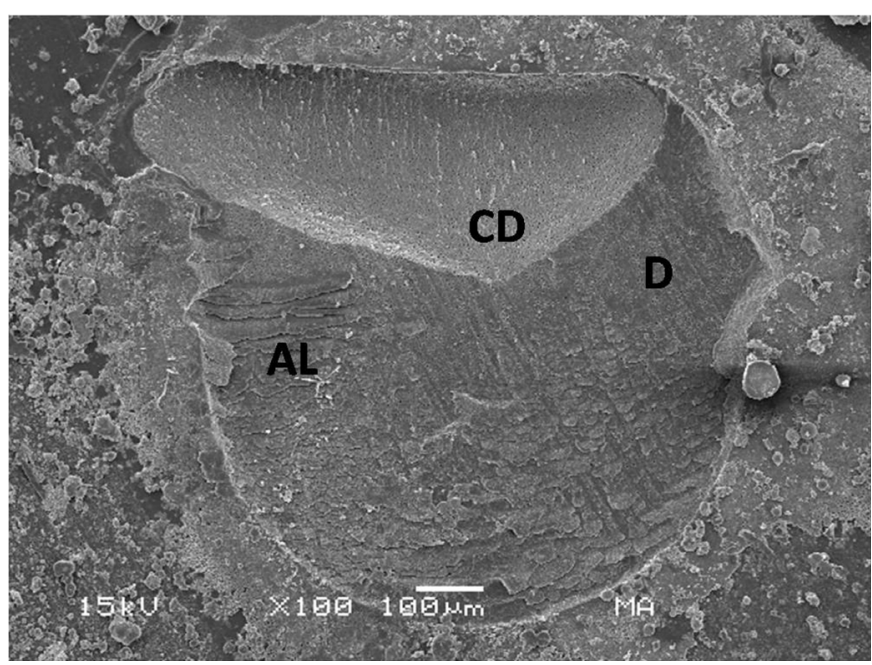

Fig. 2 - SEM photomicrograph illustrating a mixed fracture for Multilink (self-cured). Fracture within the dentin (CD) and failure between dentin and resin cement (AL: adhesive layer, D: dentin). 


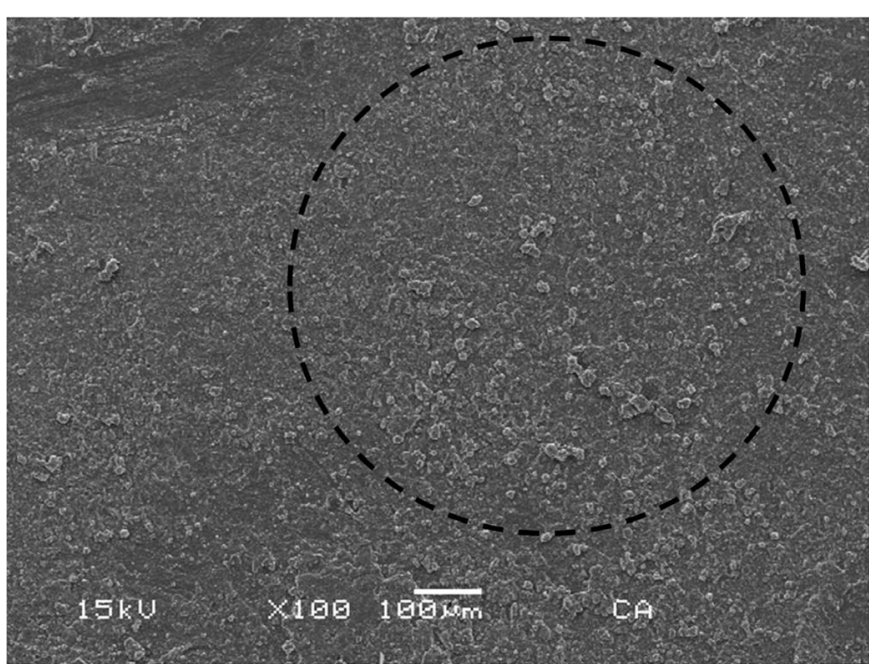

Fig. 3 - SEM photomicrograph illustrating a cohesive failure within Clearfil SA Cement (self-cured) resin cement.

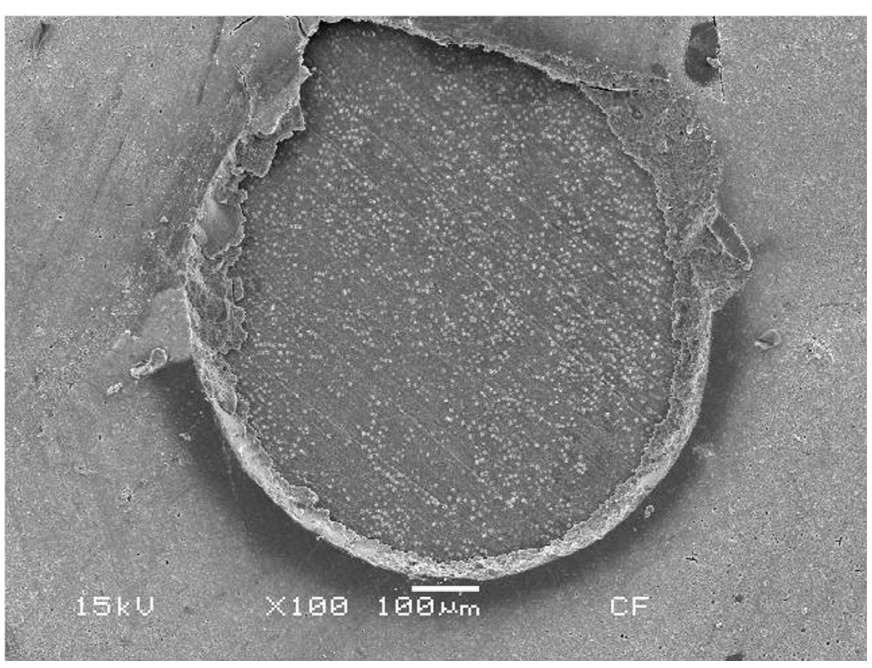

Fig. 4 - SEM photomicrograph illustrating an adhesive failure along the dentin for group Clearfil SA Cement (light-cured).

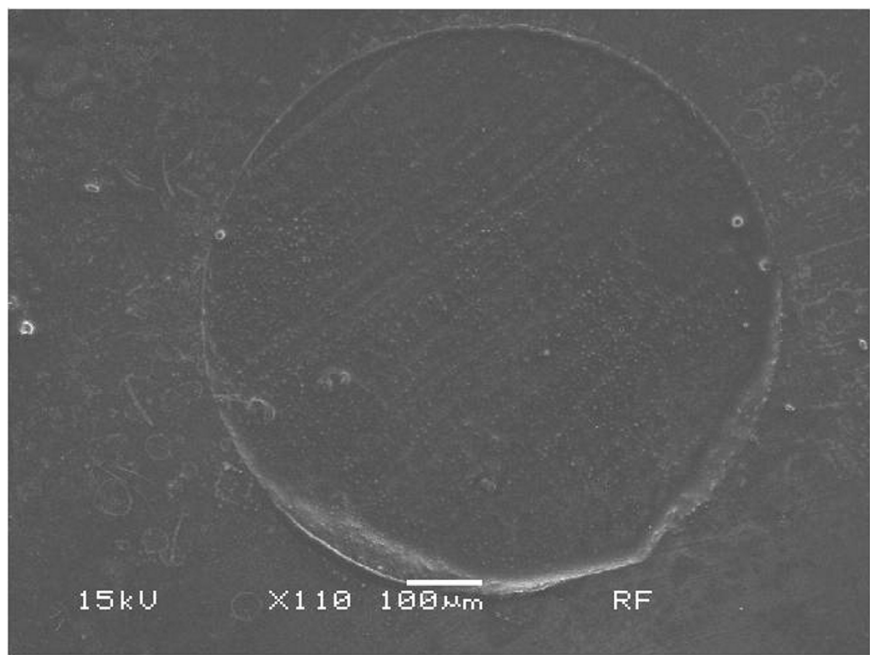

Fig. 5 - SEM photomicrograph illustrating an adhesive failure along the dentin for RelyX ARC.

\section{Discussion}

Cementation procedure plays a significant role in the clinical success of indirect restorations ${ }^{3}$. The clinical longevity of these restorations is dependent of the cavity design, the indirect restorative material, the properties of the $\mathrm{RC}$ and cementation technique selected ${ }^{9}$. The most common tests for resin-ceramic bonding measurements are shear and tensile bond strength tests ${ }^{3}$. Twenty years ago, shear and tensile BS tests were performed in specimens with relatively large bonded areas, approximately 7-28 $\mathrm{mm}^{2}$, what was questioned due to the heterogeneity of the bonded surfaces and the stress distribution at the bonded interface during the testing ${ }^{10,11}$. To overcome the non-uniform stress created in conventional shear and tensile BS tests, microshear and microtensile bond tests were developed, and these tests used specimens with a $1 \mathrm{~mm}^{2}$ cross-sectional area of approximately ${ }^{3}$. The micro-tensile and micro-shear bond strength tests were developed by Sano et al. ${ }^{12}$ (1994) and Shimada et al. ${ }^{7}$ (2002), respectively, in attempt to improve the quality of the BS measurements.

The new self-adhesives, dual-polymerizing RC were designed to simplify the cementation procedures ${ }^{13}$. These cements have appeared on the market with a proposal to simplify the adhesive bonding technique, with the use of cement in one step ${ }^{14}$, eliminating the demand for pretreatment of the tooth and the restorations. Clearfil SA Cement and Multilink resin cements are considered simplified RC, since the Clearfil SA Cement is one-step selfadhesive cement and the Multilink is applied in an association with a self-etching adhesive. Also, they are less technique-sensitive and time consuming when compared with multi-step $\mathrm{RC}^{15}$. The bonding effectiveness to dentin of these RL is dependent on quality of functional monomers from resin monomer for Clearfil SA Cement or self-etching adhesive for Multilink. The functional adhesive monomer of Clearfil SA Cement is the 10-methacryloyloxydecyl dihydrogen phosphate (10-MDP). MDP can modify the smear layer and result in demineralization of dentin ${ }^{16}$, however, in this study the self-adhesive resin monomer containing 10-MDP showed lower bond strength than Multilink and RelyX ARC cementing systems. Most of experimental groups using self-adhesive systems presented bond strength values significantly lower than those observed in the control. Other studies have also shown superior bond strength measurements for the conventional RC in comparison to the selfadhesive resin cements ${ }^{4,6,17}$.

Multilink resin cement was placed over primed dentin (Multilink Primer A + B), which contains co-initiators that can react with the acidic resin monomers to produce free radicals, initiating the reaction of self-cured $\mathrm{RC}$ and primers ${ }^{18}$. The Primer $\mathrm{A}$ is an aqueous solution of initiators, while the Primer B contains HEMA (2-hydroxyethyl methacrylates) and phosphoric acid acrylate monomers. It is possible to speculate that the presentation mode of co-initiators and the quality of bonding promoted by self-etching adhesive from Multilink cementing system were the main reasons for no differences in bond strength for the dual-polymerizing (self + light-cured) group of Multilink when compared to the control group.

The two autopolymerizing groups exhibited lower bond strength values than the dual-polymerizing groups, demonstrating that the curing mode significantly affected the bond strength. The 
overall properties of RC using the dual-cure system seem to be superior when compared to the single-cure system ${ }^{19}$. Noronha Filho et al. ${ }^{9}$ (2010) observed that RC present low degree of conversion when the indirect restoration material attenuates the light activation. The dual curing $\mathrm{RC}$ has been developed to overthrown these conditions ${ }^{20}$. The dual-cure process is due to both external light and chemical agent in RC curing. In this process occurs first the polymerization induced by light and the polymerization induced by chemical agent is performed later. This technique satisfies incomplete polymerization due to insufficient light transmittance at the bottom of the sample ${ }^{19}$.

Light activation changed the failure mode on Clearfil SA Cement. Cohesive failures within RC (Figure 3) were observed for Clearfil SA Cement without light exposure, while light activation resulted in adhesive failures (Figure 4). The low degree of conversion of this $\mathrm{RC}$ in self-cure mode reduced the bond strength to dentin and the mechanical properties, such as fracture toughness, diametric tensile strength and hardness ${ }^{21}$.

The combination of bonding agent (Scotchbond Multipurpose) and RC (RelyX ARC) was used as control in this study. De Munck et al. ${ }^{22}$ (2005) assure that a comparison of contemporary adhesives reveals that the three-step, ethanol-water-based etchand-rinse adhesives remain the 'gold standard' in terms of adhesion durability. Scotchbond Multipurpose belongs to this category of the best adhesives, which has primer and bonding agents (hydrophobic resin) in separate bottles. These categories of adhesive systems always involve the use of phosphoric acid, which permits demineralization of the dental tissues and, after rinsing, a complete elimination of the smear layer ${ }^{23}$. This category of adhesive systems is capable of reaching high degree of conversion and consequently bond strength to dentin, as showed in this study ${ }^{22}$.

Further studies will be carried out at additional times because the 24 hours may not correspond to the maximum polymerization rate, especially for the self-curing mode materials. Also, the longterm study of this subject would be important to learn more about the adhesive properties to dentin of the cementing system tested. This study used bovine teeth instead of humans, because bovine dentin morphology and adhesion are similar to human dentin and bond strength values were compatible with results published previously ${ }^{24}$.

In conclusion, light activation increased the bond strength to dentin for Clearfil SA Cement and Multilink. No significant difference in bond strength was noted between control and lightcured Multilink group. Other experimental groups showed lower bond strength to dentin than that obtained with the control.

\section{Acknowledgements}

The authors would like to thank the Capes / Brazil (Coordenação de Aperfeiçoamento de Pessoal de Nível Superior - PROCAD) for the financial support. The authors state that they have no conflicts of interest.

\section{References}

1. Belli R, Pelka M, Petschelt A, Lohbarrer V. In vitro wear gap formation of self-adhesive resin cements: a CLSM evaluation. J Dent. 2009 Dec;37(12):984-93. doi: 10.1016/j.jdent.2009.08.006.

2. Sekhri S, Mittal S, Garg S. Tensile bond strength of self adhesive resin cement after various surface treatment of enamel. J Clin Diagn Res. 2016 Jan;10(1):ZC01-4. doi: 10.7860/JCDR/2016/13409.7026.

3. Tiana T, Tsoib J K-H, Matinlinnab JP, Burrowa MF. Aspects of bonding between resin luting cements and glass ceramic materials. Dent Mater. 2014 Jul;30(7):e147-62. doi: 10.1016/j.dental.2014.01.017.

4. Irie M, Maruob Y, Nishigawab G, Suzukia K, Watts DC. Physical properties of dual-cured luting-agents correlated to early no interfacialgap incidence with composite inlay restorations. Dent Mater. 2010 Jun;26(6):608-15. doi: 10.1016/j.dental.2010.02.012.

5. Graiff L, Rasera L, Calabrese M, Vigolo P. Bonding effectiveness of two adhesive luting cements to glass fiber posts: pull-out evaluation of three different post surface conditioning methods. Int J Dent. 2014;2014:148571. doi: 10.1155/2014/148571.

6. Viotti RG, Kasaz A, Pena CE, Alexandre RS, Arrais CA, Reis AF. Microtensile bond strength of new self-adhesive luting agents and conventional multistep systems. J Prosthet Dent. 2009 Nov; 102(5):30612. doi: 10.1016/S0022-3913(09)60180-3.

7. Shimada Y, Yamaguchi S, Tagami J. Micro-shear bond strength of dualcured resin cement to glass ceramics. Dent Mater. 2002 Jul;18(5):380-8.

8. Stefani A, Brito Jr RB, Kina S, Andrade OS, Ambrosano GMB, Carvalho $A A$, et al. Bond strength of resin cements to zirconia ceramic using adhesive primers. J Prosthodont. 2016 Jul;25(5):380-5. doi: 10.1111/ jopr.12334.

9. Noronha Filho JD, Brandão NL, Poskus LT, Guimarães JGA, Silva EM. A critical analysis of the degree of conversion of resin-based luting cements. J Appl Oral Sci. 2010 Sep-Oct;18(5):442-6.

10. Armstrong S, Geraldeli S, Maia R, Raposo LH, Soares CJ, Yamagawa J. Adhesion to tooth structure: a critical review of "micro" bond strength test methods. Dent Mater. 2010 Feb;26(2):e50-62. doi: 10.1016/j. dental.2009.11.155.

11. Braga RR, Meira JBC, Boaro LCC, Xavier TA. Adhesion to tooth structure: A critical review of "macro" test methods. Dent Mater. 2010 Feb;26(2):e38-49. doi: 10.1016/j.dental.2009.11.150.

12. Sano H, Shono T, Sonoda H, Takatsu T, Ciucchi B, Carvalho R, et al. Relationship between surface area for adhesion and tensile bond strength--evaluation of a micro-tensile bond test. Dent Mater. 1994 Jul;10(4):236-40.

13. Schenke F, Federlin M, Hiller K-A, Moder D, Schmalz G. Controlled, prospective, randomized, clinical evaluation of partial ceramic crowns inserted with RelyX Unicem with or without selective enamel etching. Results after 2 years. Clin Oral Investig. 2012 Apr;16(2):451-61. doi: 10.1007/s00784-011-0516-0.

14. Silami FDJ, Tonani R, Alandia-Román CC, Pires-de-Souza FCP. Influence of different types of resin luting agents on color stability of ceramic laminate veneers subjected to accelerated artificial aging. Braz Dent J. 2016 Jan-Feb;27(1):95-100. doi: 10.1590/01036440201600348.

15. Cantoro A, Goracci C, Carvalho CA, Coniglio I, Ferrari M. Bonding potential of self-adhesive luting agents used at different temperatures to lute composite onlays. J Dent. 2009 Jun;37(6):454-61. doi: 10.1016/j. jdent.2009.02.006.

16. Liu C, Liu H, Qian Y-T, Zhu S, Zhao S-Q. The influence of four dual-cure resin cements and surface treatment selection to bond strength of fiber post. Int J Oral Sci. 2014 Mar;6(1):56-60. doi: 10.1038/ijos.2013.83.

17. Ilie N, Simon A. Effect of curing mode on the micro-mechanical properties of dual-cured self-adhesive resin cements. Clin Oral Investig. 2012 Apr;16(2):505-12. doi: 10.1007/s00784-011-0527-x.

18. Arrais CA, Giannini M, Rueggeberg FA. Effect of sodium sulfinate salts on the polymerization characteristics of dual-cured resin cement systems exposed to attenuated light-activation. J Dent. 2009 
Mar;37(3):219-27. doi: 10.1016/j.jdent.2008.11.016.

19. Kang E-S, Jeon Y-C, Jeong C-M, Huh J-B, Yun M-J, Kwon Y-H. Effect of solution temperature on the mechanical properties of dual-cure resin cements. J Adv Prosthodont. 2013 May;5(2):133-9. doi: 10.4047/ jap.2013.5.2.133.

20. Kim A-R, Jeon Y-C, Jeong C-M, Yun M-J, Choi JW, Kwon YH, et al. Effect of activation modes on the compressive strength, diametral tensile strength and microhardness of dual-cured self-adhesive resin cements. Dent Mater J. 2016;35(2):298-308. doi: 10.4012/dmj.2015056 .

21. Manso AP, Silva NR, Bonfante EA, Pegoraro TA, Dias RA, Carvalho
RM. Cements and adhesives for all-ceramic restorations. Dent Clin North Am. 2011 Apr;55(2):311-32, ix. doi: 10.1016/j.cden.2011.01.011.

22. De Munck J, Van Landuyt K, Peumans M, Poitevin A, Lambrechts $P$, Braem $M$, et al. A critical review of the durability of adhesion to tooth tissue: methods and results. J Dent Res. 2005 Feb;84(2):118-32.

23. Geerts S, Bolette A, Seidel L, Gueders A. An in vitro evaluation of leakage of two etch and rinse and two self-etch adhesives after thermocycling. Int J Dent. 2012;2012:852841. doi: 10.1155/2012/852841.

24. Reis AF, Giannini M, Kavaguchi A, Soares CJ, Line SR. Comparison of microtensile bond strength to enamel and dentin of human, bovine, and porcine teeth. J Adhes Dent. 2004 Summer;6(2):117-21. 